\title{
Line Segment Detection with Hough Transform Based on Minimum Entropy
}

\author{
Zezhong $\mathrm{Xu}^{1,2}$ and Bok-Suk Shin ${ }^{1}$ \\ 1 Department of Computer Science, The University of Auckland \\ Auckland, New Zealand \\ ${ }^{2}$ College of Computer Information Engineering, Changzhou Institute of Technology \\ Changzhou, Jiangsu, China \\ \{zxu531@aucklanduni.ac.nz, b.shin@auckland.ac.nz\}
}

\begin{abstract}
The Hough transform is a popular technique used in the field of image processing. In this paper, fitting and interpolation techniques are employed to compute high-accuracy peak parameters by considering peak spreading. The entropy is selected to measure the scatter-degree of voting. The voting in each column is considered as a random variable and voting values are considered as a probabilistic distribution. The corresponding entropies are computed and used to estimate the peak parameters. Endpoint coordinates of a line segment are computed by fitting a sine curve with more cells. It is more accurate and robust compared to solving directly two equations. The proposed method is tested on simulated and real images.
\end{abstract}

Keywords: Hough transform, peak detection, entropy, endpoints.

\section{Introduction}

The Hough transform (HT) [123] is a common technique for line segment extracting. However,image noise and parameter quantization 456] cause peak spreading problems in Hough space.

In order to deal with peak spreading and to achieve accurate peaks, researchers have proposed variations and extensions to the HT.

Some papers are focused on peak enhancement [7] in accumulator space by modifying the HT voting scheme. By assigning different weights [8] or utilizing a voting kernel [910, peaks in Hough space become more distinct and peak finding becomes easier. Other papers aim at obtaining high accuracy peak parameters, given an existing peak. Two accurate peak detection methods are presented in 1112; a smoothing window and interpolation are employed to achieve accurate peak parameters. Another high accuracy HT [13] is based on the theoretical symmetry of the butterfly.

The information-theoretic measure of entropy [141516 is often applied in image analysis, such as for clustering or pattern recognition [17, distortion correction [18, image alignment [19], or image thresholding 20]21. The peak localization method presented here is motivated by information entropy. The peak is located at $\theta$ where voting is clustered and entropy is minimal. 
The HT can also be used for extracting complete parameters of a line segment in an image although the HT provides only distance $\rho$ and the angle $\theta$.

Some methods provide a complete line-segment description using the HT. One kind of methods is based on image space 22 23 24. After applying the standard HT, the line is broken into disjoint segments in image space. The endpoints of a line segment are determined by taking the projection of the feature points on either the $x$ - or $y$-axis. Two thresholds are needed for the length of line segment and gaps between line segments. Another kind of methods is based on the Hough space. By analysing the butterfly distribution [25|26] around a peak in the accumulator array, butterfly features are used to discover parameters of a line segment. The first and last non-zero values are obtained and used to compute the endpoints of the line segment by solving two sets of simultaneous equations [27 28 29:30. However, how to find the first and the last non-zero voting value is a difficult problem.

This paper focuses on improving accuracy and robustness of peak detection. By considering various uncertainties, the voting in each column is considered as a random variable and voting values are considered as a probabilistic distribution. Corresponding entropies are computed and used to estimate the peak parameter. Endpoint coordinates of a line are computed by fitting a sine curve with upside and downside cells in columns around a peak rather than solving two equations generated from just two cells.

The rest of the paper is organized as follows. Section 2 describes our peak detection method based on minimum entropy. Section 3 outlines endpoint detection by fitting sine curves. Section 4 compares methods by providing experimental results. Section 5 concludes.

\section{Peak Detection}

After voting all pixels in an image for all possible cells into Hough space, the next step is peak detection. Considering the peak spreading, we locate the peak where the voting is the most clustered.

\subsection{Voting Distribution around a Peak}

Under an ideal situation, voting distribution around a peak is symmetrical and convergent into a focal cell in the middle column. In each column around a peak, the sum of voting values is a constant; see Fig. 1. A peak can be found and computed easily in this case.

However, due to various uncertainties, like image noise, quantization noise, or line thickness, cells around an actual peak are usually not symmetrical and there is not a focal cell. In each column around a peak, the sum of voting values is constant only approximately; see Fig. 2 Voting is scattered in $\rho$ direction in some $\theta$ columns. The degree of voting scattered or clustered is different in the columns. The final peak is located where the voting is the 'most clustered'. 


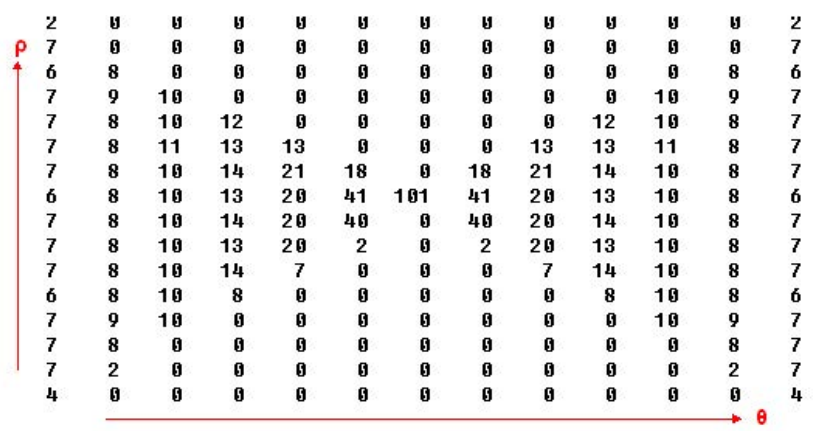

Fig. 1. An ideal peak region

\begin{tabular}{|c|c|c|c|c|c|c|c|c|c|c|c|c|c|c|c|}
\hline 10 & 8 & 16 & 6 & 2 & 1 & 1 & 1 & 4 & 3 & 1 & 1 & (6 & 3 & 10 & 9 , \\
\hline$\rho 7$ & 8 & 8 & 18 & 5 & B & 1 & 2 & 2 & 2 & B & 1 & 1 & 8 & 11 & 9 \\
\hline 410 & 10 & 13 & 8 & 15 & 5 & 1 & 2 & 3 & 1 & 2 & 1 & 8 & 13 & 11 & 10 \\
\hline 7 & 11 & 9 & 14 & 12 & 16 & 3 & 5 & 1 & 2 & 2 & 3 & 13 & 12 & 10 & 11 \\
\hline 12 & 12 & 10 & 12 & 15 & 16 & 19 & 3 & 3 & 2 & 2 & 17 & 15 & 16 & 13 & 8 \\
\hline 6 & 7 & 8 & 8 & 14 & 21 & 25 & 13 & 1 & 1 & 18 & 20 & 21 & 13 & 14 & 15 \\
\hline 13 & 10 & 13 & 12 & 13 & 17 & 21 I & 35 & 19 & 21 & 30 & 29 & 21 & 18 & 13 & 10 \\
\hline 10 & 10 & 10 & 12 & 14 & 16 & 28 & 40 & 79 & 70 & 46 & 26 & 19 & 18 & 13 & 9 \\
\hline 7 & 11 & 10 & 14 & 18 & 19 & 17 & 34 & 52 & 68 & 36 & 21 & 16 & 10 & 11 & 12 \\
\hline 10 & 8 & 14 & 15 & 12 & 15 & 24 & 28 & 14 & 5 & 31 & 28 & 16 & 11 & 12 & 13 \\
\hline 5 & 11 & 12 & 12 & 13 & 16 & 23 & 11 & อ & 1 & 3 & 18 & 18 & 17 & 11 & 9 \\
\hline 11 & 7 & 8 & 9 & 17 & 20 & 10 & 3 & B & B & 3 & 7 & 15 & 14 & 13 & 9 \\
\hline 6 & 9 & 7 & 18 & 15 & 15 & 5 & B & 1 & 1 & B & 3 & 11 & 14 & 11 & 11 \\
\hline 6 & 9 & 17 & 7 & 15 & 3 & 4 & 1 & 1 & g & 1 & 1 & 3 & 9 & 12 & 9 \\
\hline 8 & 13 & 7 & 16 & 2 & 3 & 1 & 5 & 1 & 1 & 0 & 2 & 2 & 4 & 10 & 8 \\
\hline 13 & 6 & 11 & 3 & 4 & 2 & 3 & 3 & 3 & 1 & 3 & 1 & B & 2 & 5 & 12 \\
\hline 6 & 9 & 7 & 3 & 1 & 1 & 2 & 1 & 3 & 4 & 1 & o & 1 & 1 & B & 4 \\
\hline
\end{tabular}

Fig. 2. An actual peak region

\subsection{Entropy of Voting}

In information theory [14, if $X$ is a discrete random variable with distribution $P\left(X=x_{k}\right)=p_{k} \quad k=1,2, \cdots$ then the entropy of $X$ is defined as follows:

$$
E(X)=-\sum p_{k} \log p_{k}
$$

The entropy is used to measure the uncertainty of the probability distribution. The smaller the entropy $E$ is, the larger the cluster-degree is. Voting in each column is considered to be a random variable; entropy can be used to measure the voting scattering. In $\theta_{i}$ column in the peak region, the probability of voting for a cell $H_{i j}(\theta, \rho)$ is computed as $p_{i j}=H_{i j}(\theta, \rho) / \Sigma H_{i j}(\theta, \rho)$ where $j$ belongs to a peak region. The entropy of the $\theta_{i}$ column is then

$$
E_{i}=-\sum p_{i j} \log p_{i j}
$$

Because voting distributions differ in different column, the $E_{i}$-values for columns also differ. The more clustered a voting is, the smaller the entropy. The more scattered a voting is, the bigger the entropy. A lower entropy distribution means that voting is clustered and can be considered as a peak. By minimizing information entropy of voting, an optimal $\theta$ peak is detected. 


\subsection{Peak $\theta$ Computation}

Around a peak region, we perform the following operations: (1) Compute the entropy $E_{i}$ of each $\theta_{i}$ column according to Equ. (2). Fig. 3 shows the entropy $E$ of each $\theta$ column. (2) Fitting a curved function $f$.

$$
f: E=f(\theta)
$$

(3) Compute $\theta_{\text {peak }}$ where the fitted function has a minimum $\theta_{\text {peak }}=\theta \mid f^{\prime}(\theta)=0$.

\subsection{Peak $\rho$ Computation}

Around a peak region, we also do: (1) Compute means

$$
m_{i}=\sum\left[H_{i j}(\rho, \theta) \cdot \rho_{j}\right] / \sum H_{i j}(\rho, \theta)
$$

of each $\theta$ column. (2) A sinusoidal curve fitting. Sine curve is a nonlinear function. Fitting directly a sine curve is difficult, so we fit it by taking advantage of a linear function. In the equation of standard HT $\rho=x \cdot \cos \theta+y \cdot \sin \theta$, the relation between $\rho$ and $\theta$ is not linear. It can be linear by using the following transform:

$$
\rho / \cos \theta=y \cdot \tan \theta+x
$$

$\rho / \cos \theta$ and $\tan \theta$ are in linear relation. With all these $\left(m_{i} / \cos \theta_{i}, \tan \theta_{i}\right)$, a linear function is fitted. The RANSAC technique is employed by considering various uncertainties:

$$
g: \rho / \cos \theta=g(\tan \theta) \triangleq b_{1} \tan \theta+b_{0}
$$

(3) Interpolation to compute $\rho_{\text {peak }}$ corresponding to $\rho_{\text {peak }}$ :

$$
\rho_{\text {peak }}=b_{0} \cos \left(\theta_{\text {peak }}\right)+b_{1} \sin \left(\theta_{\text {peak }}\right)
$$

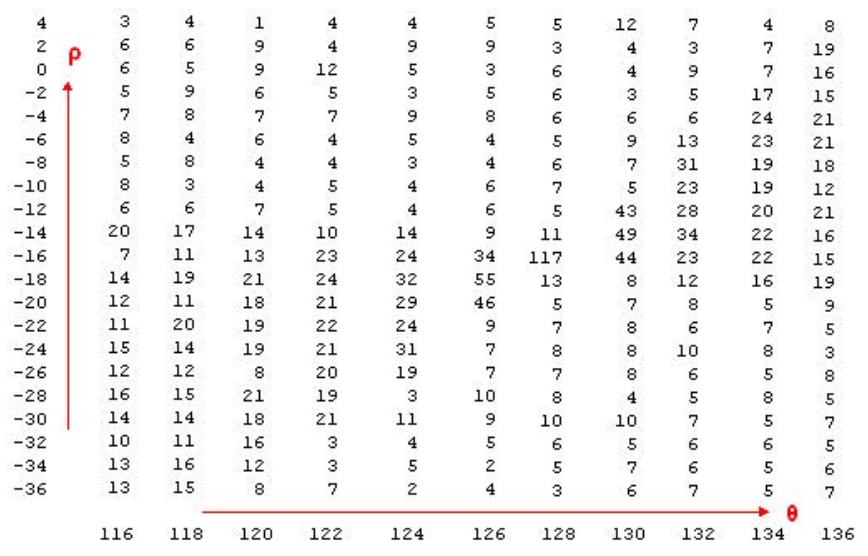

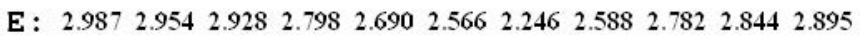

Fig. 3. Entropy of each column around a peak 


\subsection{Multiple Line Detection}

The existence of multiple lines in the given image leads to overlays of multiple peaks in Hough space. If two peaks are far away, peak detection is only a little influenced because the final peak parameters are fitted and interpolated. If a peak region overlaps partly with other peak regions, the voting values and entropies in the overlapped columns become bigger. These columns are neglected for fitting and interpolation.

After a peak $\left(\rho_{\text {peak }}, \theta_{\text {peak }}\right)$ is detected, the voting values corresponding to this peak are removed in order not to preclude the detection of another peak. First, with $\left(\rho_{\text {peak }}, \theta_{\text {peak }}\right)$, those pixels contributing to this peak are selected in the image space. Second, the votes of those pixels are inverted by decreasing value of corresponding cells by 1 .

\section{Endpoint Detection}

We find the endpoint coordinates of a line segment, called left endpoint $p\left(x_{l}, y_{l}\right)$ and right endpoint $p\left(x_{r}, y_{r}\right)$. Left and right is defined by the origin (i.e. the perpendicular point). See Fig. 4. According to the equation of the HT, if we have two cells $\left(\theta_{1}, \rho_{1}\right)$ and $\left(\theta_{2}, \rho_{2}\right)$ where a left endpoint votes for, the coordinates of the left endpoint can be computed directly by solving two simultaneous equations

$$
\begin{aligned}
& \rho_{1}=x \cdot \cos \theta_{1}+y \cdot \sin \theta_{1} \\
& \rho_{2}=x \cdot \cos \theta_{2}+y \cdot \sin \theta_{2}
\end{aligned}
$$

By considering various uncertainties, rather than solving directing two equations, we compute the coordinates of endpoints by fitting more cells.

\subsection{Valid Cell Identification}

Due to parameter quantization and image noise, a peak region is not distinct in Hough space. It is usually as in Fig. 5. Cells corresponding to endpoints can not be determined simply by identifying zero and non-zero voting values, which is used in many methods for extracting endpoints.

The collinear pixels and image noise all vote for the cells in a peak region. The voting values from collinear pixels are considered as foreground, while the voting

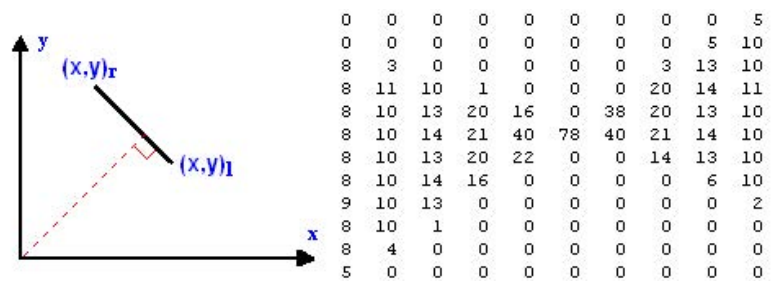

Fig. 4. Left endpoint and right endpoint 


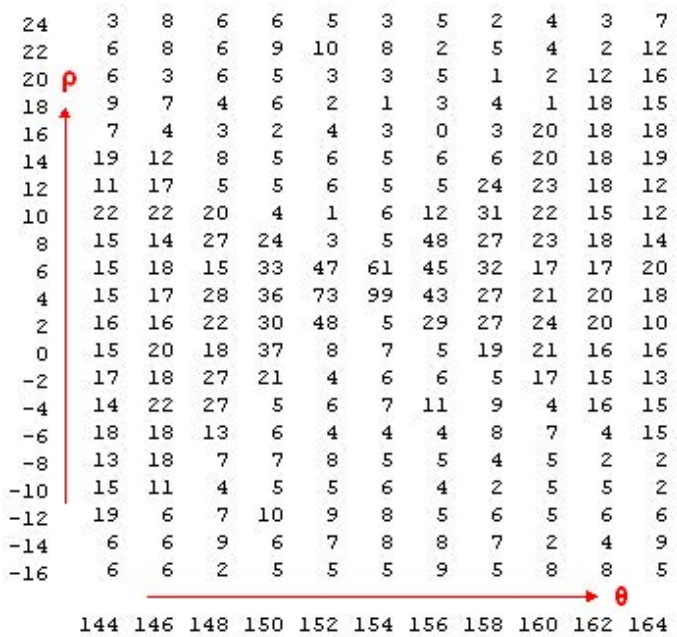

Fig. 5. Peak region before background separation

values from noise or other pixels are considered as background. A threshold is determined to divide cells in a peak region into background and foreground cells. In each column, a threshold is determined by a maximum inter-class variance method. Thus, background cells are separated. A peak region after background separation is shown in Fig. 6]

\subsection{Endpoint Coordinate Computation}

Consider foreground cells in the left column of the peak; upside cells are selected to compute the right endpoint and downside cells are selected to compute the

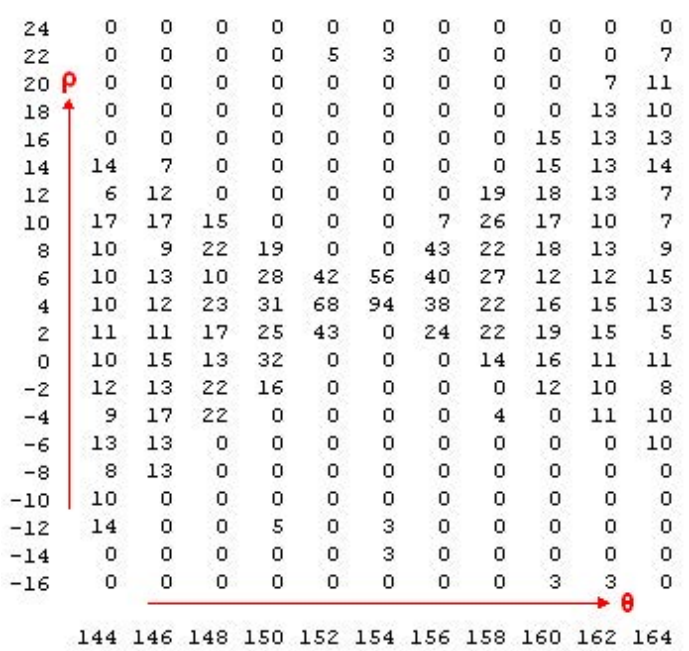

Fig. 6. Peak region after background separation 
left endpoint. While in the right column of a peak, upside cells and downside cells are respectively used to compute right and left endpoint. Using all cell parameters $\left(\theta_{i}, \rho_{i}\right)$ for which the left endpoint votes, a sine curve is fitted.

As discussed above, $\rho / \cos \theta$ and $\tan \theta$ are in a linear relationship. With all these $\left(\rho_{i} / \cos \theta_{i}, \tan \theta_{i}\right)$, a linear function is fitted:

$$
g: \rho / \cos \theta=g(\tan \theta) \triangleq b_{1} \tan \theta+b_{0}
$$

Endpoint coordinates are then as follows: $x_{l}=b_{0}$ and $y_{l}=b_{1}$. Similarly, the right endpoint can be computed by fitting cell parameters $\left(\rho_{i}, \theta_{i}\right)$ for which the right votes.

\section{Experimental Results}

In this section, the proposed peak detection and endpoint extracting methods are applied to a set of simulated data to test the accuracy of line detection, and applied to real world image to verify the performance of line-segment detection.

\subsection{Tests with Simulated Images}

We generate $M \times N=200 \times 200$ binary images that contain randomly generated noisy pixels. Endpoint coordinates of one line segment are also produced randomly, and line parameters $(\theta, \rho)$ are computed and recorded as ground truth.

We test the methods in term of different counts of noisy pixels, different quantization, or different line thickness. Fig. 7 shows the detection error under ideal conditions. Fig. 8 shows the detection error when parameter quantization, line thickness and image noise are comprehensively considered.
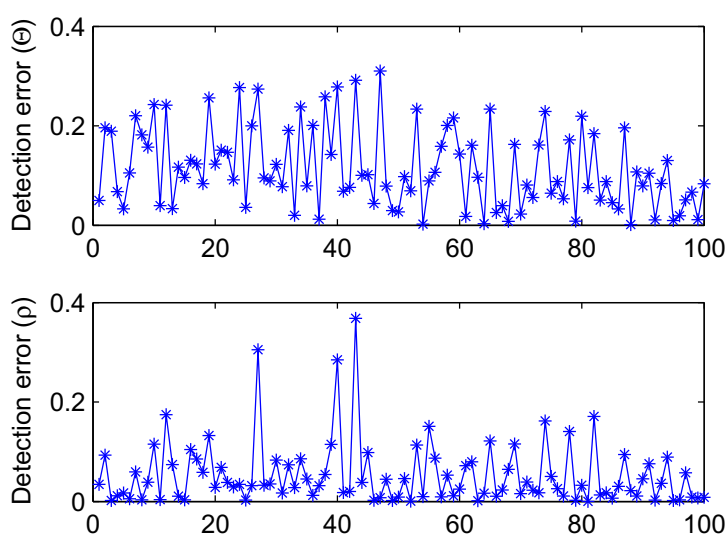

Fig. 7. Test under ideal conditions. Count of noise: 0, parameter quantization: 1, line thickness: 0 . 

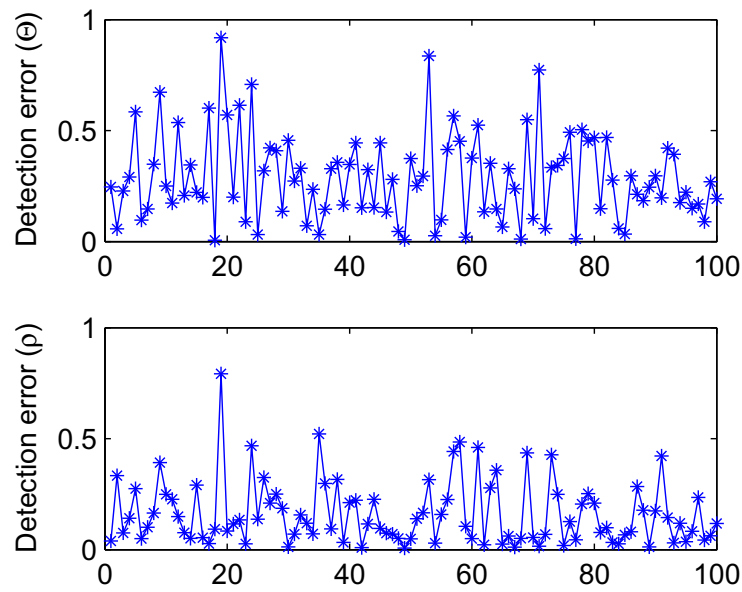

Fig. 8. Comprehensive test. Count of noise: 500, parameter quantization: 2 , line thickness: 2 .

The detection results from the proposed peak detection method based on minimum entropy $(M E)$ are compared with results of a method using smoothing and interpolating $(S E I I)$ [12, and with results of least-squares fitting $(L S F) .100$ random line segments are tested in terms of the absolute error with respect to the ground truth. Detection results are shown in Fig. 9, where the count of noise is 500 , parameter space is quantized as $(2,2)$, and line thickness is 2 .
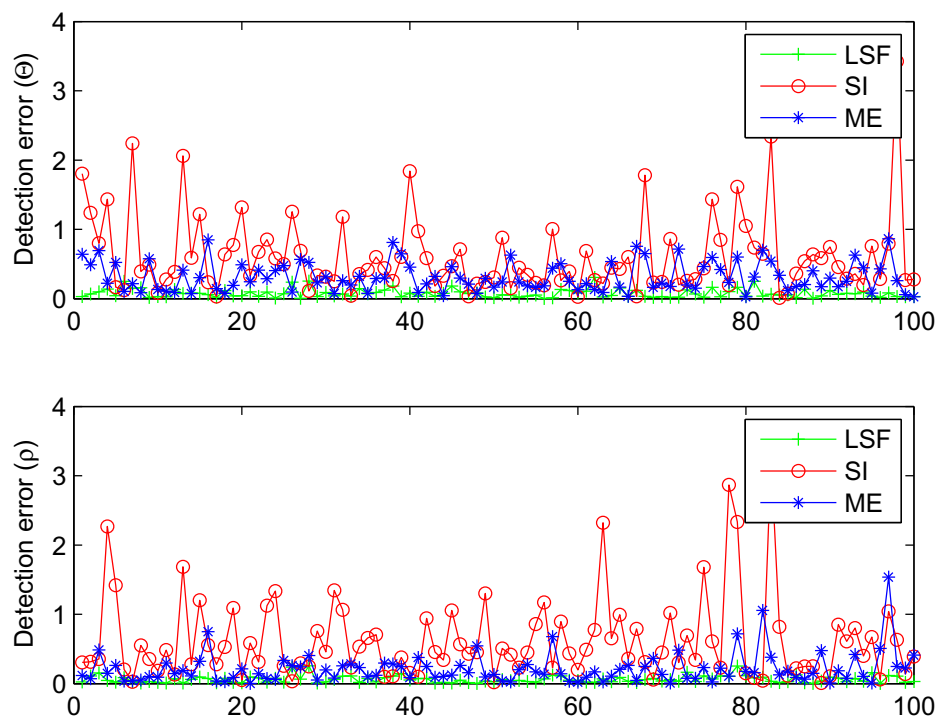

Fig. 9. Comparison of detection errors 


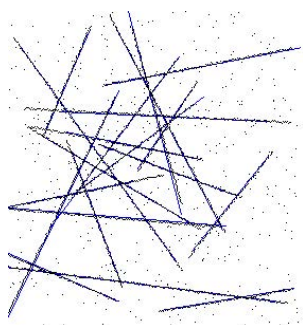

Fig. 10. Line segment detection in simulated images

With the proposed method based on minimum entropy, sub-cell accuracy is achieved. Detection results with the proposed method are close to the results of least-square fitting, even under coarse parameter quantization and image noise.

\subsection{Endpoint Tests}

A simulated image, which contain 500 random noise pixels and 20 random line segments, is used to test the proposed method. The parameter space is quantized with $(\Delta \theta, \Delta \rho)=(2,2)$ and thickness of line segment is 2 . Detection results are shown in Fig. 10, All line segments are detected in spit of image noise, coarse parameter quantization and line thickness.

\subsection{Real World Tests}

We use crystalline graphite fibre images 31 to test the proposed method for extracting line segments. A detection result is shown in Fig. 11, in this image only the 50 longest line segments are extracted. Although these fibre images are very complicated, many line segments are detected by identifying valid cells and fitting a sine curve with more cells parameters.
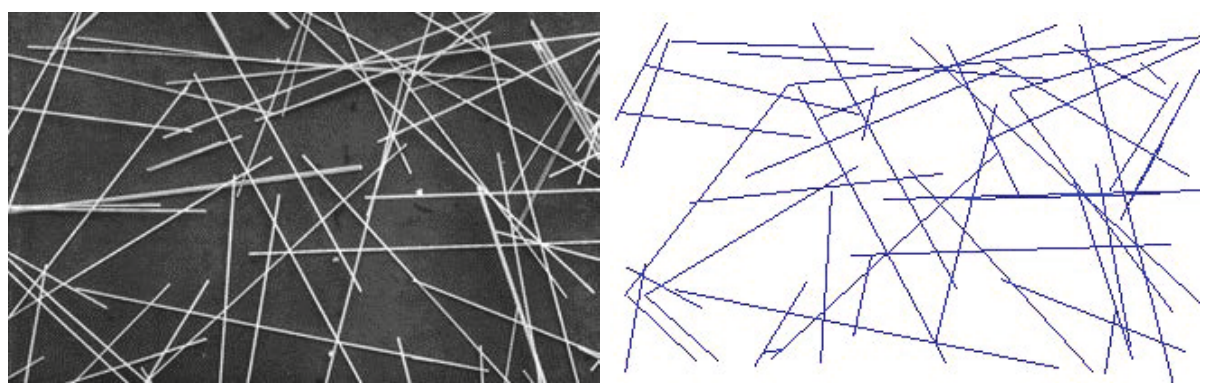

Fig. 11. Line segment detection for fibre image. Left: Original image. Right: Detected line segments. 


\section{Conclusions}

A line segment detection method with Hough transform is proposed. The method for the distance and angle detection is based on minimum entropy and has been compared with other methods. Using fitting and interpolating techniques based the minimum entropy, the Hough transform can yield very accurate peak parameters that are close to results from least-squares fitting for simulated images. The method for endpoints extraction applies fitting a sine curve. It is more accurate and robust compared with methods that solve simultaneous equations.

Acknowledgments. The authors would like to thank Reinhard Klette for his comments and kind support. The first author thanks Jiangsu Overseas Research \& Training Program for University Prominent Young \& Middle-aged Teachers and Preside for granting a scholarship to visit and undertake research at The University of Auckland.

\section{References}

1. Hough, P.V.C.: Methods and means for recognizing complex patterns, U.S. Patent 3.069.654 (1962)

2. Duda, R.O., Hart, P.E.: Use of the Hough transformation to detect lines and curves in pictures. Comm. ACM. 15(1), 11-15 (1972)

3. Ballard, D.H.: Generalizing the Hough transform to detect arbitrary shapes. Pattern Recognition 13(2), 111-122 (1981)

4. Zhang, M.: On the discretization of parameter domain in Hough transform. In: Proc. IEEE ICPR 1996, pp. 527-531 (1996)

5. Lam, W.C.Y., Lam, L.T.S., Yuen, S.Y., Leung, D.N.K.: An analysis on quantizing the hough space. Pattern Recognition Letters 15(10), 1127-1135 (1994)

6. Van Veen, T.M., Groen, F.C.A.: Discretization errors in the Hough transform. Pattern Recognition 14, 137-145 (1981)

7. Ji, J., Chen, G., Sun, L.: A novel Hough transform method for line detection by enhancing accumulator array. Pattern Recognition Letters 32(11), 1503-1510 (2011)

8. Guo, S., Pridmore, T., Kong, Y., Zhang, X.: An improved Hough transform voting scheme utilizing surround suppression. Pattern Recognition Letters 30, 1241-1252 (2009)

9. Fernandes, L.A.F., Oliveira, M.: Real-time line detection through an improved Hough transform voting scheme. Pattern Recognition 41(1), 299-314 (2008)

10. Palmer, P.L., Kittler, J., Petrou, M.: An optimizing line finder using a Hough transform algorithm. Computer Vision Image Understanding 67(1), 1-23 (1997)

11. Niblack, W., Petkovic, D.: On improving the accuracy of the Hough transform: Theory, simulation, and experiments. In: IEEE Conf. Computer Vision Pattern Recognition, pp. 574-579 (1988)

12. Niblack, W., Petkovic, D.: On improving the accuracy of the Hough transform. Machine Vision Applications 3, 87-106 (1990)

13. Du, S., Tu, C., Sun, M.: High accuracy Hough transform based on butterfly symmetry. Electron Letters 48(4), 199-201 (2012) 
14. Shannon, C.E.: The mathematical theory of communication. The Bell System Tech. J. 27, 379-423 (1948)

15. Titterington, D.M.: The maximum entropy method for data analysis. Nature 312(38), 1-382 (1984)

16. Yuan, L., Kesavan, H.K.: Minimum entropy and information measure. IEEE Trans. Syst., Man, Cybern. 28(3), 488-491 (1998)

17. Watanabe, S.: Pattern recognition as a quest for minimum entropy. Pattern Recognition 13(5), 381-387 (1981)

18. Rosten, E., Loveland, R.: Camera distortion self-calibration using the plumb-line constraint and minimal hough entropy. Machine Vision Applications 22(1), 77-85 (2011)

19. Zhu, D., Wang, L., Yu, Y., Tao, Q., Zhu, Z.: Robust ISAR Range Alignment via Minimizing the Entropy of the Average Range Profile. IEEE Geoscience Remote Sens. Lett. 6(2), 204-208 (2009)

20. Brink, A.: Using spatial information as an aid to maximum entropy image threshold selection. Pattern Recognition Letters 17(1), 29-36 (1996)

21. Abutaleb, A.S.: Automatic thresholding of gray-level pictures using two dimensional entropy. Computer Vision Graphics and Image Processing 47, $22-32$ (1989)

22. Yamato, J., Ishii, I., Makino, H.: Highly accurate segment detection using Hough transformation. Systems and Computers in Japan 21(1), 68-77 (1990)

23. von Gioi, R.G., Jakubowicz, J., Morel, J.M., Randall, G.: On Straight Line Segment Detection. Journal of Mathematical Imaging and Vision 32(3), 313-347 (2008)

24. Nguyen, T.T., Pham, X.D., Jeon, J.: An improvement of the standard Hough transform to detect line segments. In: Proc. IEEE Int. Conf. Ind. Technology, pp. $1-6(2008)$

25. Furukawa, Y., Shinagawa, Y.: Accurate and robust line segment extraction by analyzing distribution around peaks in Hough space. Computer Vision Image Understanding 92(1), 1-25 (2003)

26. Du, S., Tu, C., van Wyk, B.J., Ochola, E.O., Chen, Z.: Measuring straight line segments using HT butterflies. PLoS One 7(3), e33790 (2012)

27. Atiquzzaman, M., Akhtar, M.W.: Complete line segment description using the hough transform. Image Vision Comp 12(5), 267-273 (1994)

28. Atiquzzaman, M., Akhtar, M.W.: A robust Hough transform technique for complete line segment description. Real-Time Imaging 1(6), 419-426 (1995)

29. Kamat, V., Ganesan, S.: A Robust Hough Transform Technique for Description of Multiple Line Segments in an Image. In: Proceedings of 1998 International Conference on Image Processing (ICIP 1998), vol. 1, pp. 216-220 (1998)

30. Kamat-Sadekar, V., Ganesan, S.: Complete description of multiple line segments using the Hough transform. Image and Vision Computing 16(9-10), 597-613 (1998)

31. http://www.ilo.orgoshencimagesstoriesenlargedPart01RES_imgsRES200F2 